INTERNAL

MAMMARY ARTERY

FLOW REGULATION

\title{
The pressure flow relationship of the internal mammary artery after offpump anastomosis to the left anterior descending coronary artery
}

Mark B. Connellan, Stephanus J. Roos, André R. Coetzee and Gawie J. Rossouw

Departments of Cardiothoracic Surgery, Anaesthesiology and Critical Care, Tygerberg Hospital, University of Stellenbosch, South Africa

Address for correspondence:

Department of Cardiothoracic Surgery

Faculty of Medicine

PO Box 19213

Tygerberg

7505

South Africa

Email:

mark_connellan@hotmail.com

\section{INTRODUCTION}

The internal mammary artery (IMA) is the most commonly used conduit graft for anastomosis to the left anterior descending (LAD) coronary artery, being used on average $80 \%$ of the time.(I) Offpump coronary artery bypass grafting (OPCAB) remains a commonly employed strategy for revascularisation with many reported benefits, ${ }^{(2,3,4)}$ despite recent evidence to dispute this. ${ }^{(5)}$ This study aimed to assess the changes in flow of the internal mammary artery relative to varying arterial blood pressures, directly after $O P C A B$ grafting of the left internal mammary artery to the LAD. The pressure flow relationship was also assessed with the IMA in situ and after having skeletonising the artery.

We hypothesised that there may be a loss of autoregulation once the IMA was removed from the chest wall and after anastomosis to the IMA. We tested this hypothesis using three different anaesthetic techniques, using halothane, propofol and sevoflurane. For the purpose of this study, autoregulation refers to the perfusion pressure-coronary blood flow relationship and we did not attempt to incorporate the third variable of autoregulation i.e. work of the heart, into the study.

\section{ABSTRACT}

Objective: To assess the pressure flow relationship of the internal mammary artery (IMA) in situ, after skeletonisation and after anastomosis to the left anterior descending (LAD) coronary artery, using either halothane, sevoflurane or propofol as an anaesthetic agent.

Methods: 15 Pigs were used in total, five received halothane, five sevoflurane and five propofol as an anaesthetic agent. The flow in the internal mammary artery in each of the pigs, was measured at various arterial pressures. This was done with the IMA in situ, then after dissecting the artery off the chest wall using the skeletonisation technique and finally after offpump grafting to the left anterior descending coronary artery.

Results: The pressure flow relationship of the internal mammary artery after skeletonisation was found to be linear $(r=0.8650)$. The pressure flow correlation after grafting the skeletonised internal mammary artery to the left anterior descending coronary artery was found to be similarly linear $(r=0.8766)$. In the sevoflurane subgroup, with the IMA still in situ, a degree of autoregulation was found to be present, but after skeletonisation this was subsequently lost $(\mathrm{p}=0.0 \mathrm{II})$.

Conclusions: The pressure flow relationship in the internal mammary artery after skeletonising the vessel and after OPCAB anastomosis to the LAD was found to be linear. In the subgroup of pigs receiving sevoflurane, some degree of autoregulation was demonstrated in the in situ IMA. This remnant of autoregulation was lost after skeletonisation and after grafting of the vessel to the left anterior descending coronary artery. SAHeart 2009; 6:222-228

\section{MATERIALS AND METHODS}

This study was conducted with the permission of the Ethics Committee of the University of Stellenbosch, Faculty of Health Sciences, and animals were cared for in accordance with institutional and international guidelines.

In order to generate enough experimental units, we planned the initial use of 15 pigs. Although an initial calculation of required sample size was not done, the data resulted in generating a statis- 
tical power of $>0.95$ for the three IMA groups, and $>0.80$ for the anaesthetic subgroups. Upon completion of this, sufficient statistical significance was established and further experimentation would not have likely added further value to the study.

The 15 pigs used for the analysis weighed $23-39 \mathrm{~kg}$ and were of both sexes. The pigs were premedicated with intramuscular ketamine $(5 \mathrm{mg} / \mathrm{kg})$. Intravenous access was established via an ear vein and the animals received $25 \mathrm{ml} / \mathrm{kg} / \mathrm{hr}$ Ringer's lactate for the remainder of the procedure. Anesthesia was induced with pentobarbital $(8 \mathrm{mg} / \mathrm{kg})$, thiopental $(2 \mathrm{mg} / \mathrm{kg}$ ) and fentanyl (I 6ug/ $/ \mathrm{kg})$. A tracheostomy was performed after loss of eyelid reflex, the trachea was intubated and the animals ventilated (Julian, Drager, Germany) with $50 \%$ oxygen and air. The tidal volume was set at $10 \mathrm{ml} / \mathrm{kg}$ and the $\mathrm{PaCO} 2$ maintained between $34-36 \mathrm{mmHg}$. Muscle relaxation was achieved with pancuronium bromide, using an initial bolus dose of $0.2 \mathrm{mg} / \mathrm{kg}$ followed by a constant infusion of $0.3 \mathrm{mg} / \mathrm{kg} / \mathrm{h}$.

Five of the pigs were randomly allocated to receive halothane for maintenance of anaesthesia at $0.7 \%$ expiratory concentration. A further five randomly assigned pigs received propofol as a constant intravenous infusion according to the Bristol protocol.(6) A three compartment pharmacokinetic computer model simulation using this protocol, predicted a blood level of $2.5-3.0 \mathrm{ug} / \mathrm{ml}$. This is similar to levels achieved in the clinical setting. The remaining five pigs received sevoflurane at $2 \%$ expiratory concentration for maintenance of anaesthesia.

A neck dissection was performed and a stiff 16 gauge catheter inserted into the carotid artery and advanced to within $3 \mathrm{~cm}$ of the aortic valve. This was connected to a pressure transducer (BASSEM504T, Biometrix, The Netherlands) and used to continuously display aortic systolic, diastolic and mean arterial pressures. ECG leads were placed and lead I was continuously monitored (Datex-Ohmeda, General Electric, USA).

A median sternotomy was performed. A $\mathrm{Icm}$ segment of the left IMA was mobilised free from the surrounding tissue. A size 12 electromagnetic flow probe ( $T$ I06 Small Animal Blood Flow Meter, Transonic, USA) was placed on the IMA. Mean arterial pressure (MAP) was varied by positional manipulation of the pig.
Varying amounts of Trendelenberg and anti-Trendelenberg positioning resulted in obtaining a range of mean arterial pressures from 26-1 I $4 \mathrm{mmHg}$. Flow through the artery was then measured at 3-8 different mean arterial blood pressures after allowing for stabilisation of flow for 5 minutes at that specific pressure.

The left IMA was dissected out in a skeletonised fashion in its entirety: from its origin at the subclavian artery to its distal bifurcation. The artery was not ligated or divided. The arterial pressure was again manipulated by positioning of the pig in the manner described above and flow in the skeletonised IMA was measured at various pressures. The pig was then heparinised $(250 \mathrm{u} / \mathrm{kg}$ intravenously) and the left internal mammary artery divided distally. No vasodilator agent was used on the IMA.

The pericardium was then suspended. A deep pericardial stay suture was placed between the left superior and inferior pulmonary veins to aid in positioning of the heart. The LAD coronary artery was stabilised using a tissue stabiliser (Octopus Evolution, Medtronic, USA) and the LAD was proximally snared. The LAD was opened and during a brief period of snaring, an appropriate sized shunt (ClearView Intracoronary Shunt, Medtronic, USA) was inserted into the coronary artery. The proximal snare was released and the left internal mammary artery was then anastomosed in an end-to-side fashion to the LAD on the beating heart.

After completion of the anastomosis, the proximal coronary artery was snared. Arterial pressure was positionally manipulated and a third set of flow measurements through the IMA was recorded. On completion of the experiment, the grafts were opened and inspected to ensure patency. In order to assess the relative pressure and flow relationship in each of the experimental steps, the percentage of maximal recorded flow of the IMA was compared to the percentage of maximal arterial blood pressure measured.

\section{Statistical methods used}

Since different pressures were applied to the IMA to measure the $\%$ flow under different treatments given to the pigs, the internal mammary artery was considered to be the experimental unit. Linear regression models were fitted with \% flow as the response variable and \% mean arterial pressure as the indepen- 
dent or concomitant variable. A significance level of alpha $=5 \%$ was used for the study. The comparison of the slopes of the lines were done with analysis of variance methods as published by Quinn and Keough. ${ }^{(7)}$

\section{RESULTS}

\section{The complete group}

The mean baseline MAP for the group as a whole was $67 \mathrm{mmHg}$. The relationship between flow and pressure after grafting the IMA to the LAD was found to be linear $(r=0.8766)$. Similarly the relationship between flow and pressure after skeletonising the IMA was found to be linear $(r=0.8650)$. The pressure flow relationship of the in situ IMA was also found to be linear in nature $(r=0.7863)$. This calculated regression line was numerically more horizontal than the skeletonised and anastomosed lines, but the gradients (slopes) of these lines did not differ significantly $(F=2.395, p=0.093)$. This relationship is shown in Figure $\mathrm{I}$.

\section{The halothane subgroup}

The mean baseline MAP before positional manipulation in this group was $57 \mathrm{mmHg}$ (range of baseline pressures $41 \mathrm{mmHg}$ to $80 \mathrm{mmHg}$ ). The induced pressures and resulting flows generated for each pig are presented in Figure 2. The observed relationships between pressure and flow were linear for the in situ IMA

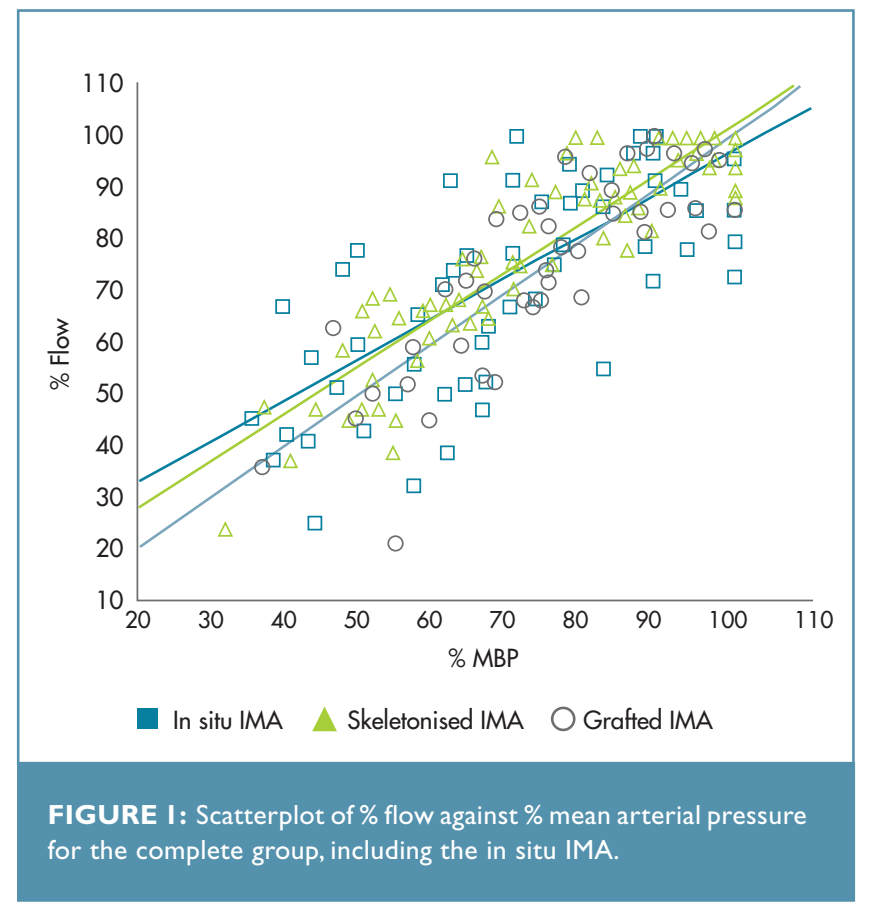

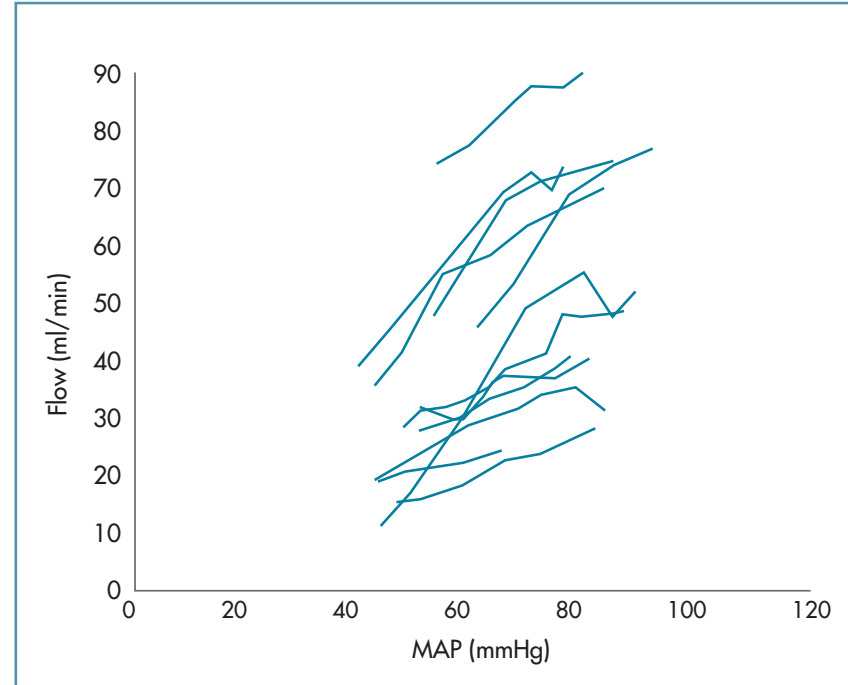

FIGURE 2: Line graph of flow ( $\mathrm{ml} / \mathrm{min})$ against mean arterial pressure $(\mathrm{mmHg})$ for the in situ, skeletonised and anastomosed IMA for pigs receiving halothane anaesthesia.

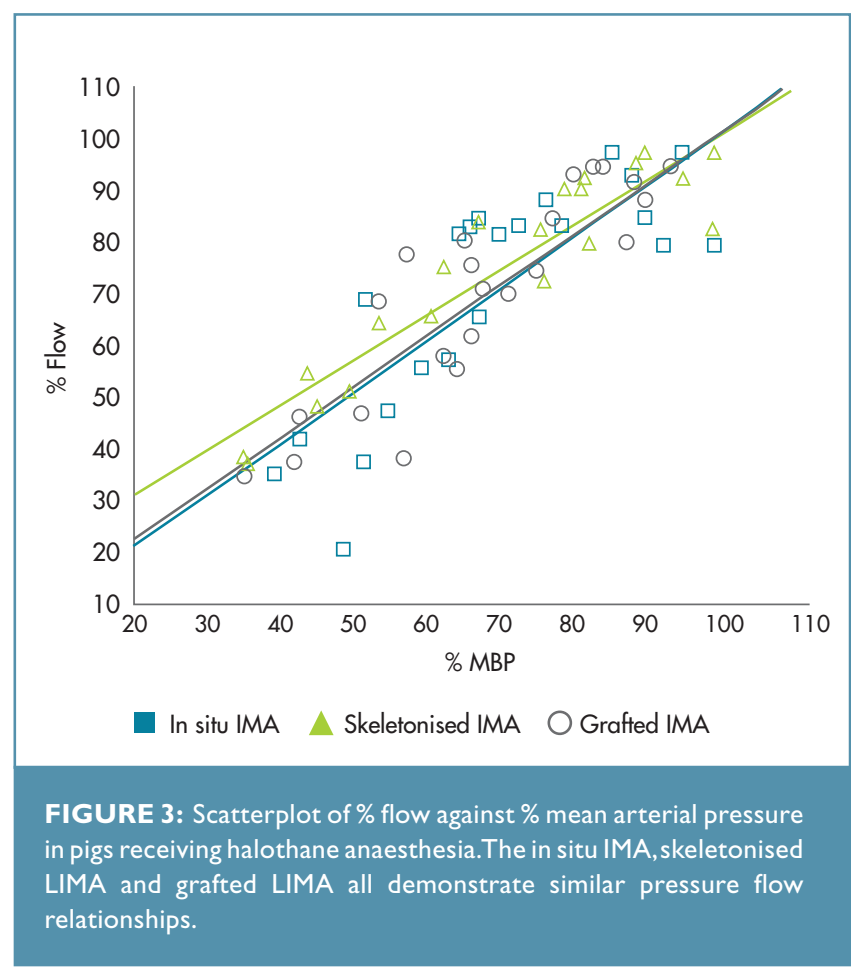

$(r=0.8553)$, the skeletonised IMA $(r=0.939$ I) and the grafted IMA $(r=0.9129)$. There was no significant difference in the gradients of the regression lines as depicted in Figure 3. This suggests that there is no autoregulation of the IMA during halothane anaesthesia.

The propofol subgroup

The mean baseline MAP before positional manipulation in the 


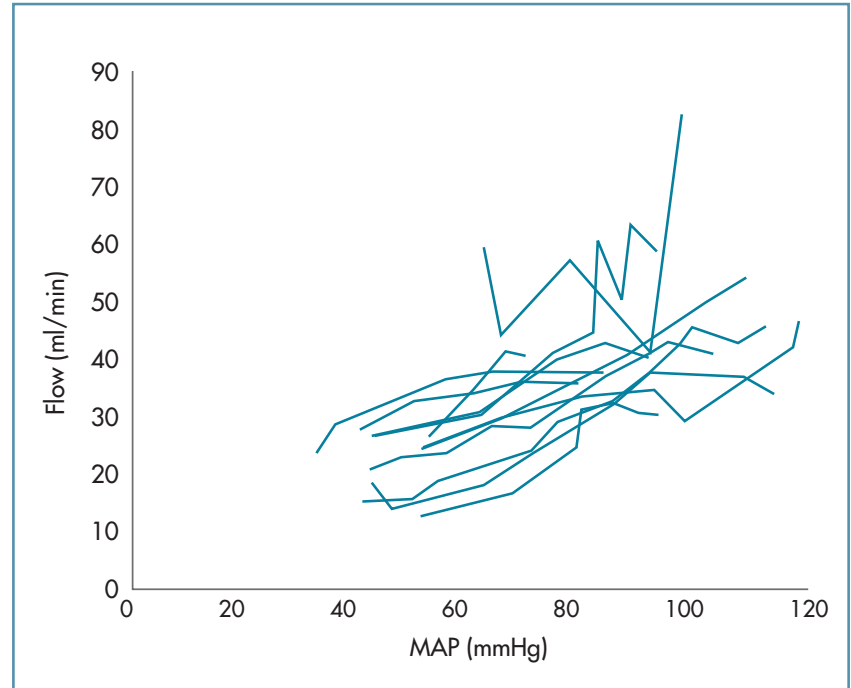

FIGURE 4: Line graph of flow $(\mathrm{ml} / \mathrm{min})$ against mean arterial pressure $(\mathrm{mmHg})$ for the in situ, skeletonised and anastomosed IMA for pigs receiving propofol anaesthesia.

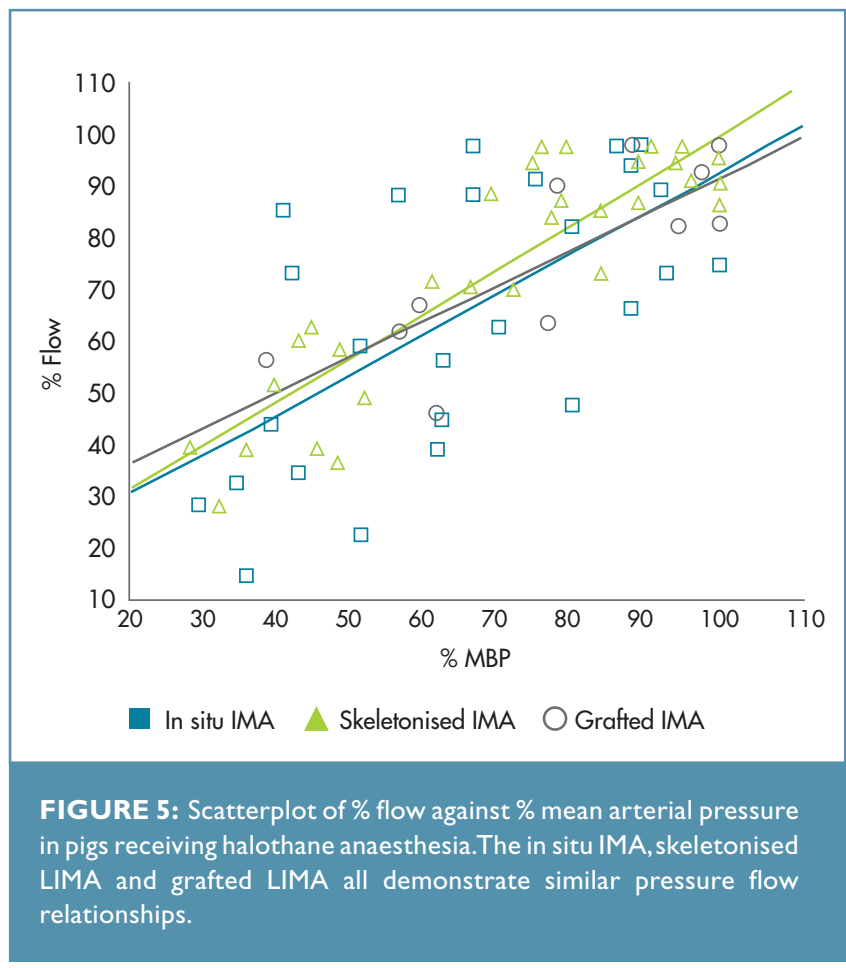

group receiving propofol was $76 \mathrm{mmHg}$ (range of baseline pressures $39 \mathrm{mmHg}$ to $119 \mathrm{mmHg}$ ). The induced pressures and resulting flows generated for each pig in this group, are presented in Figure 4. The relationships in this subgroup were similar to the halothane subgroup. The in situ IMA had a linear pressure flow relationship $(r=0.7089)$. The skeletonised IMA and the grafted IMA also demonstrated linear relationships $(r=0.9064$ and $r=0.8103$ respectively). No significant difference in the gradients of these regression lines was seen in this subgroup as seen in Figure 5. As with the halothane subgroup, this suggests that there is no autoregulation of the IMA during propofol anaesthesia.

\section{The sevoflurane subgroup}

The sevoflurane group had a mean baseline MAP of $68 \mathrm{mmHg}$ (range of baseline pressures $29 \mathrm{mmHg}$ to $102 \mathrm{mmHg}$ ). The induced pressures and resulting flows generated for each pig for the sevoflurane group, are presented in Figure 6. The in situ IMA demonstrated a linear pressure flow relationship $(r=0.8799)$ in this group. A linear correlation between pressure and flow of the skeletonised and grafted IMA was also found in this subgroup ( $r=0.9189$ and $r=0.8869$ respectively). However, the regression line gradients differed significantly, $(F=4.852, p=0.0 \mathrm{I}$ I) with the skeletonised and grafted IMA lines more vertical than the in situ IMA line. Figure 7 depicts these findings. These results suggest that the in situ IMA exhibited some degree of flow autoregulation (compared to the skeletonised and anastomosed IMA). This remnant of autoregulation is however lost upon skeletonisation of the IMA and after anastomosis to the LAD.

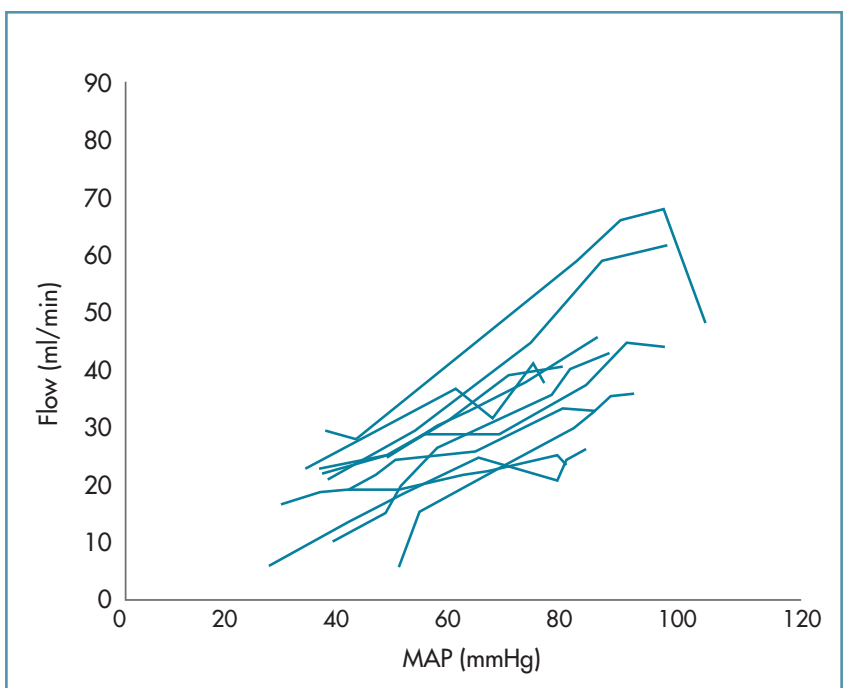

FIGURE 6: Line graph of flow $(\mathrm{ml} / \mathrm{min})$ against mean arterial pressure $(\mathrm{mmHg})$ for the in situ, skeletonised and anastomosed IMA for pigs receiving sevoflurane anaesthesia. 


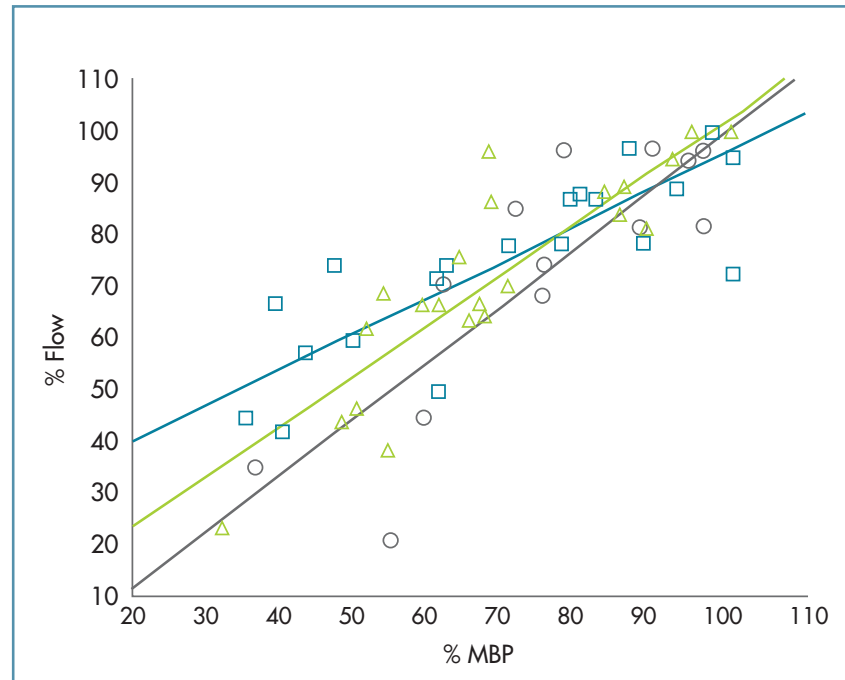

In situ IMA $\triangle$ Skeletonised IMA $\bigcirc$ Grafted IMA

FIGURE 7: Scatterplot of \% flow against \% mean arterial pressure in pigs receiving sevoflurane anaesthesia. The skeletonised LIMA and grafted LIMA have a linear relationship which is significantly more vertical than that of the in situ IMA pressure flow correlation $(F=4.852, p=0.01 \mathrm{I})$. This suggests some loss of autoregulatory mechanisms controlling flow in the in situ IMA.

\section{DISCUSSION}

We have demonstrated that in a porcine model, the flow of blood through the IMA is directly dependent on the arterial pressure. A similar linear relationship between pressure and flow exists in venous coronary grafts. This passive pressure flow relationship was present once the IMA was skeletonised and, for the group as a whole, was already present in the in situ IMA. No significant change in pressure flow relationship of the IMA, from in situ to after skeletonisation and after anastomosis, was found in the subgroups receiving halothane or propofol.

Some evidence of a degree of autoregulation in the in situ IMA could only be demonstrated in the pigs receiving sevoflurane. In this subgroup, the regression line depicting the in situ pressure flow relationship was significantly more horizontal than in the IMA groups after skeletonising and grafting. In the sevoflurane group, a degree of flow regulation seen in the in situ IMA has thus shown to be reduced by skeletonising the IMA. The mechanism for this is not clearly defined. By skeletonising the IMA, sympathetic unmyelinated nerve fibers supplying the muscular layers of the vessel, are likely damaged. ${ }^{(9)}$ This may result in a decrease in the amounts of norepinephrine and vasoactive peptide which are normally released by these nerve endings. $(10,11)$ The lack of these mediators may result in a decrease in the autoregulatory potential of the IMA to vasoconstrict, as the normal balance with vasodilators such as nitric oxide (NO), prostacyclin and endothelium-derived hyperpolarising factor is disturbed. $(12,13)$

There is also evidence that skeletonising the IMA may lead to dysfunctional or denuded arterial endothelium. ${ }^{(14)} \mathrm{NO}$, normally synthesised in the endothelial layer, is known to be largely responsible for arterial conduit vasodilation in response to an increase in blood flow. Dysfunctional endothelium, which can arise from skeletonising the IMA, may thus lead to a decrease in the autoregulatory capacity of the artery to vasodilate. The relationships of these vasoactive agents are complex and the relative contribution of each agent under varying conditions is not known. Sevoflurane causes relaxation of vascular smooth muscle in a concentration dependant manor, mainly through a decrease in the intracellular calcium concentration. ${ }^{(16)}$ This has been experimentally shown to occur via a direct inhibition of calcium release from the sarcoplasmic reticulum or sarcolemmal calcium stores. ${ }^{(17)}$ Evidence also exists that sevoflurane decreases the sensitising of contractile proteins by calcium, however the exact mechanism is not fully understood. ${ }^{(18)}$ In addition sevoflurane inhibits the second messenger IP3 which is responsible for calcium release intracellularly. ${ }^{(19)}$ It does this to a lesser extent than either halothane or propofol. Sevoflurane may thus potentially inhibit autoregulatory vasoconstriction by decreasing the vascular tone of the in situ IMA, and this may well explain the absence of normal autoregulation in the IMA as shown by our results.

Halothane like sevoflurane inhibits intracellular calcium concentration as well as contractile apparatus sensitivity. ${ }^{(20)}$ The effect of lowering intracellular calcium is more evident at lower concentrations, and the desensitising of contractile elements more pronounced at higher concentrations of the agent.(20) Convincing evidence exists that the decrease of intracellular calcium concentration is through inhibition of release from the sarcoplasmic stores. $^{(21)}$ Halothane is also known to inhibit the L-type calcium release channels, thereby decreasing plasmalemmal calcium influx, further contributing to decrease in contractile ability. ${ }^{(22)}$ Halothane, unlike sevoflurane has however been shown to cause ryanodine receptor mediated release of calcium from intracellular stores. ${ }^{(23)}$ This facilitates a transient smooth muscle cell contraction via 
increased intracellular calcium. The net effect is however one of vasodilation, particularly at higher concentrations. A complex set of interactions is thus likely responsible for the absent autoregulatory mechanisms of the in situ IMA demonstrated in the halothane group.

Propofol has been shown to increase basal vascular tone at clinical and low concentrations, but decrease basal tone in arteries at high concentrations. ${ }^{(24,25)}$ This increase in basal tone seems to be endothelium independent, and is thought to be via activation of voltage dependant calcium channels. ${ }^{(26)}$ Propofol has also been shown to directly inhibit calcium release from the sarcoplasmic reticulum, as well as decreasing inositol phosphate production. ${ }^{(27,28)}$ This results in a decrease in vascular tone, however sensitised contractile elements result in a net sustained increase in smooth muscle contraction. As with halothane, a complex set of interactions is thus likely responsible for the absence of autoregulation of the in situ IMA seen in the propofol group.

Spasm of the IMA is another factor which may influence flow through the vessel after skeletonisation and grafting. This would likely result in various low flow data points upon analysis. The $r$ values of 0.8650 and 0.7863 of the skeletonised and anastomosed IMA groups, denote a reasonably good linear correlation and therefore suggest that spasm did not have a significant role to play in our experimental model. In the clinical scenario spasm of the IMA is also a possibility. The pressure flow relationship (with the possibility of a degree of spasm) we demonstrated in the porcine model, is thus also likely to be applicable to the patient.

The absence of IMA and saphenous vein graft( ${ }^{(8)}$ flow autoregulation after coronary artery bypass graft (CABG), has important implications in the clinical setting. The coronary flow, after CABG, is thus directly reliant on the perfusion pressure. After coronary surgery variation in blood pressure, especially low blood pressure, is a constant risk. The necessary usage of protamine contributes to the possibility of hypotension because of its vasodilatory properties. ${ }^{(29,30)}$ However, unlike in patients with normal coronary arteries, patients post CABG cannot rely on autoregulatory mechanisms to maintain their coronary flow. Once the blood pressure falls, either the ventricle becomes ischaemic, leading to ischaemic paralysis of the myocardium, or hibernation of the myocardium occurs, resulting in a decrease in myocardial work. ${ }^{(31,32)}$
Both mechanisms result in a decrease in cardiac output, and a negative spiral of falling blood pressures and poor cardiac output commences. A decrease in coronary flow is however offset by decrease in ventricular work, hence the risk of myocardial ischaemia is not easy to define.

We elected not to incorporate the variable of myocardial work into our study, as this would have added a significant resource intensive complexity to the study, without necessarily adding further value to the results. This decision may be partially justified by the observation that we did not find a significant difference in the slopes of the graphs of the skeletonised IMA compared to the anastomosed IMA. This would suggest that (within the limits of our study) the variation in myocardial work only played a limited role in modulating the IMA flow after it was anastomosed to the LAD. Nevertheless the exclusion of work from our study design does represent a limitation to this research.

We have demonstrated a linear correlation between pressure and flow in the IMA after skeletonising the vessel and after OPCAB anastomosis to the LAD. A degree of flow regulation of the in situ IMA in the sevoflurane subgroup were shown to be present, while no such autoregulatory mechanisms were demonstrated in the halothane or propofol subgroup.

Although care should be taken in transposing data from a porcine model to humans, our data suggest there is perhaps a direct pressure-flow relationship in IMA graft in humans. Practitioners should take cognisance of these relationships, and consider adjusting perfusion pressure to adequately increase the graft (and coronary) flow in order to meet myocardial demand. Naturally, the nature of a decrease in blood pressure should first be defined. In addition, the practitioner should consider that a similar scenario exists for on-pump coronary grafting and also in the immediate postoperative period, although we did not specifically examine these hypotheses.

\section{ACKNOWLEDGEMENTS}

The authors would like to thank Mr. Johan van Rensburg and Mr. Isaac Petersen for their valuable assistance in the animal laboratory. We would further like to thank Professor D Nel from the Centre for Statistical Consultation at Stellenbosch University, for the statistical analysis of the study data. 


\section{REFERENCES}

I. Bonchek LI. LIMA use in the STS database: a correction. Ann Thorac Surg 2002;73:345-346.

2. Halkos ME, Puskas JD, Lattouf OM, et al. Impact of pre-operative neurologic events on outcomes after coronary artery bypass grafting. Ann Thorac Surg 2008:86:504-10.

3. Puskas JD, Williams WH, Duke PG, et al. Off-pump coronary artery bypass grafting provides complete revascularization with reduced myocardial injury, transfusion requirements, and length of stay: a prospective randomized comparison of two hundred unselected patients undergoing off-pump versus conventional coronary artery bypass grafting. J Thorac Cardiovasc Surg 2003;125: 797-808.

4. Sedrakyan A, Wu AW, Parashar A et al. Off-pump surgery is associated with reduced occurrence of stroke and other morbidity as compared with traditional coronary artery bypass grafting: a meta-analysis of systematically reviewed trials. Stroke 2006;37:2759-69.

5. Chu D, Bakaeen FG, Dao TK, et al. On-pump versus off-pump coronary artery bypass grafting in a Cohort of 63000 patients Ann Thorac Surg 2009;87: 1820-7.

6. Roberts FL, Dixon J, Lewis GT, et al. Induction and maintenance of propofol anaesthesia. A manual infusion scheme. Anaesthesia 1988; 43 (suppl): |4-17.

7. Quinn GP, Keough MJ. Experimental design and data analysis for biologists. Cambridge University Press 2007.

8. Coetzee CJ. The pressure-flow relationship in venous coronary bypass grafts in the immediate post-cardiopulmonary bypass period. MMed thesis presented at the Faculty of Medicine, University of Stellenbosch. 1995

9. Deja MA, Gołba KS, Malinowski M et al. Skeletonization of internal thoracic artery affects its innervation and reactivity. Eur J Cardiothorac Surg. 2005;28:55।-7.

10. Feigl EO. Neural control of coronary blood flow. J Vasc Res 1998;35:85-92.

II. Huang AH, Feigl EO. Adrenergic coronary vasoconstriction helps maintain uniform transmural blood flow distribution during exercise. Circ Res 1988;62: 286-98.

12. Duffy SJ, Castle SF, Harper RW, et al. Contribution of vasodilator prostanoids and nitric oxide to resting flow, metabolic vasodilation, and flow-mediated dilation in human coronary circulation. Circulation 1999;100:1951-7.

13. Mombouli JV, Vanhoutte PM. Endothelial dysfunction: from physiology to therapy J Mol Cell Cardiol 1999;31:61-74.

14. Del Campo C, Pedicled or Skeletonized? A Review of the Internal Thoracic Artery Graft. Tex Heart Inst I 2003;30:170-5.

15. Rubanyi GM, Romero JC, Vanhoutte PM. Flow-induced release of endotheliumderived relaxing factor. Am J Physiol 1986;250:1 145-9.

16. Akata T, Izumi K, Nakashima M. The action of sevoflurane on vascular smooth muscle of isolated mesenteric resistance arteries: II. Mechanisms of endotheliumindependent vasorelaxation. Anesthesiology 2000;92: I 44 I-53.

17. Nakamura K, Toda H, Hatano Y, et al. Comparison of the direct effects of sevoflurane, isoflurane and halothane on isolated canine coronary arteries. Can J Anaesth 1993;40:257-61.

18. Nakamura K, Terasako K, Toda $H$, et al. Mechanisms of inhibition of endotheliumdependent relaxation by halothane, isoflurane, and sevoflurane. Can J Anaesth 1994:41:340-6

19. Yu J, Tokinaga Y, Ogawa K, et al. Sevoflurane inhibits angiotensin II-induced, protein kinase C-mediated but not Ca2_-elicited contraction of rat aortic smooth muscle. Anesthesiology 2004; 100:879-84.

20. Akata T, Boyle WA III. Dual actions of halothane on intracellular calcium stores of vascular smooth muscle. Anesthesiology 1996;84:580-95.
21. Akata T, Nakashima M, Izumi K. Comparison of volatile anesthetic actions on intracellular calcium stores of vascular smooth muscle: Investigation in isolated systemic resistance arteries. Anesthesiology 2001;94:840-50.

22. Buljubasic N, Rusch NJ, Marijic J, et al. Effects of halothane and isoflurane on calcium and potassium channel currents in canine coronary arterial cells. Anesthesiology 1992;76:990-8.

23. Su JY, Tang L-J. Effects of halothane on the sarcoplasmic reticulum Ca2_ stores and contractile proteins in rabbit pulmonary arteries. Anesthesiology 1998; 88:1096-106.

24. Imura N, Shiraishi $\mathrm{Y}$, Katsuya $\mathrm{H}$, et al. Effect of propofol on norepinephrineinduced increases in [Ca2_] in and force in smooth muscle of the rabbit mesenteric resistance artery. Anesthesiology 1998;88:1566-78.

25. Yamanoue T, Brum JM, Estafanous FG. Vasodilation and mechanism of action of propofol in porcine coronary artery. Anesthesiology 1994:81:443-51.

26. Hamada H, Damron DS, Murray PA. Intravenous anesthetics attenuate phenylephrine-induced calcium oscillations in individual pulmonary artery smooth muscle cells. Anesthesiology 1997;87:900-7.

27. Tanabe K, Kozawa $O$, Kaida T, et al. Inhibitory effects of propofol on intracellular signaling by endothelin-I in aortic smooth muscle cells. Anesthesiology 1998; 88:452-60.

28. Xuan YT, Glass PS. Propofol regulation of calcium entry pathways in cultured AIO and rat aortic smooth muscle cells. Br J Pharmacol 1996; 1 17:5-12.

29. Welsby IJ, Newman MF, Phillips-Bute B, et al. Hemodynamic changes after protamine administration: association with mortality after coronary artery bypass surgery. Anesthesiology 2005; 102 (2):308-314.

30. Michaels IA, Barash PG. Hemodynamic changes during protamine administration. Anesth Analg 1983; 62:831-5.

31. Slezak J, Tribulova N, Okruhlicova L, et al. Hibernating myocardium: pathophysiology, diagnosis, and treatment. Can J Physiol Pharmacol. 2009;87:252-65.

32. Royster RL. Myocardial dysfunction following cardiopulmonary bypass: recovery patterns, predictors of inotropic need, theoretical concepts of inotropic administration. J Cardiothorac Vasc Anesth. 1993;7(4 Suppl 2): 19-25. 